\title{
The Mechanism and Function Study of the Main Controller in HVDC Transmission System
}

\author{
P.Z. Li \& L.G. Ban \& H.W. Wang \\ China Electric Power Research Institute, Beijing, China
}

\begin{abstract}
In order to analyze the differences between HVDC system and DC system, this paper states the mechanism and function of the main controller in HVDC. Also the impact of each controller and regulator on the system is analyzed. The result shows that there are some key parameters in each controller, which determine the operation state of the system. We should consider the parameters according to the system condition.
\end{abstract}

KEYWORD: HVDC System, Control System, Control Logic, System Stability

\section{INTRODUCTION}

The energy resources and economy of China are far from each other. So it has been a kind of trend that we transports power from the western energy base to eastern region through long-distance and largecapacity power transmission. Thus, as a kind of long-distance and large-capacity power transmission technology, HVDC is an effective way to realize the optimal allocation of energy in China.

HVDC system has larger capacity, higher voltage and higher current. Its control and protection system is similar to $\pm 800 \mathrm{kV}$ HVDC system. But we need to adjust its control strategies and parameters in different $\mathrm{AC}$ and $\mathrm{DC}$ fault conditions to ensure the right action of DC system controller and maintain the stable operation after the AC fault.

\section{GENERAL STATE OF DC CONTROL SYSTEM}

DC control system is a complicated system. The operator sets the value of the direct current power and calculates the current command through the calculation of the current instruction. Power instructions generated by the added DC control function will be added after it is given by the operator, such as the change of power modulation function output and frequency control function output. The output of current instruction calculation is also restricted by overload limiter. It gets the final current order after the low-voltage current-limiting aspects. As the input of the current regulator and AMAX regulator, the voltage order if voltage regulator is generated by voltage calculation. These three main regulator are restricted to the others, and eventually generates the angle order.

\section{THE MAIN CONROLLER OF DC CONTROL SYSTEM}

\subsection{Current controller}

Rectifier and inverter are configured with the same closed-loop current regulator. By removing a current margin of the current order at the inverter side, we can control the current of the rectifier. The main objective of the closed-loop current regulator is to ensure the performance of the current controller, fast step response, steady state error of zero current, stable current control, and rapid inhibition of overcurrent fault.

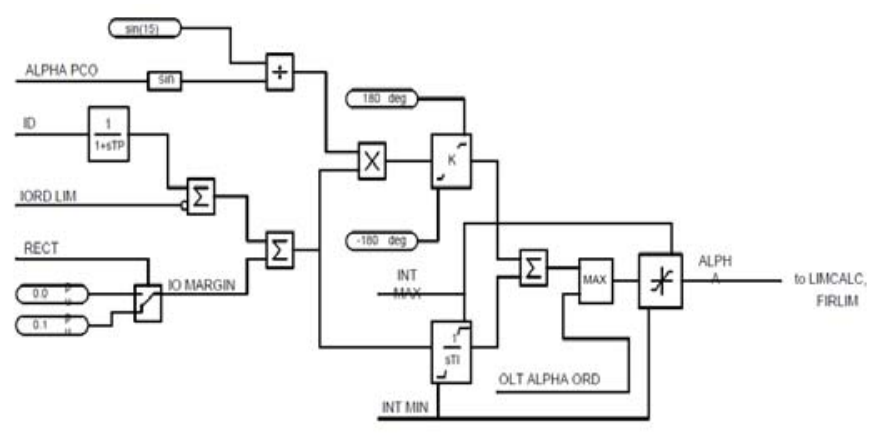

Figure 1 Control logic of current controller

The current difference of the actual DC current measurement of closed-loop current control between the current order generated by the VDCOL control- 
ler, goes through a proportional integral link, the output of which is the angle order. Figure 1 shows the control logic of current regulator.

\subsection{Voltage controller}

DC voltage controller at inverter side converts the DC voltage within the allowable range. The reference value of DC voltage is calculated by the VARC. There are controllers at both inverter side and rectifier side. The voltage controller is a PI controller. Its output will be used as the upper limit or lower limit of the current controller. When it runs as the inverter, its output will be the upper limit to limit the maximum of the current controller; when it runs as rectifier, its output will be the lower limit to limit the minimum output of the current controller.

\subsection{The maximum firing angle (AMAX) Regulators}

According to the formula of the DC voltage,

$$
U_{d}=U d i 0 *\left(\cos \gamma-\left(d_{x}-d_{r}\right)-\frac{I_{0}}{I_{d N}} * \frac{U d i o N}{U d i 0}\right)
$$

For a constant angle $\gamma$, when the DC current increases, the inverter DC voltage decreases. Therefore, when the inverter station is operating in a given $\gamma$ control, it turns out a negative resistance at low frequency.

The DC voltage of inverter could also be shown by the formula:

$$
U_{d}=\frac{1}{2} * U_{d i 0} *(\cos \gamma+\cos (\gamma+\mu))
$$

Commutation resistance $\mathrm{d}_{\gamma}$ only affect the DC voltage. It does not affect the length of the phase change time. When we merge two formulas above, and ignore the commutation resistance $\mathrm{d}_{\gamma}$, we can get:

$$
\beta=(\mu+\gamma)=\arccos \left(\cos \gamma-2 * d_{x} * \frac{I_{0}}{I_{d N}} * \frac{U_{d i 0 N}}{U_{d i 0}}\right)
$$

According to the calculated $\beta$ angle by the formula above, the inverter can run at a given $\gamma$-side state. The inverter rated $\gamma$ angle is generally $17^{\circ}$.

If we adjust the formula with a current term for the given $\gamma$ control, we can make it a positive slope at the transient state, which helps to improve the stability of the system.

$\beta=(\mu+\gamma)=\operatorname{arcco}\left(\cos \gamma-2 * d_{X} * \frac{I_{0}}{I_{d N}} * \frac{U_{d i 0 N}}{U_{d i 0}}-K\left(I_{0}-I_{d}\right)\right)$

Under steady-state conditions, $I_{d}$ is equal to $I_{0}$. The angle $\beta$ given by the formula above can make inverter run at a given $\gamma$ state. At the transient state, when Io remain unchanged and $I_{d}$ increases due to disturb- ance, AMAX controller will decrease angle $\beta$ to make the voltage higher and the DC current lower. So the system could goes back to the steady state; On the contrary, when $\mathrm{I}_{\mathrm{d}}$ becomes smaller because of the disturbance, AMAX controller will increase the angle $\beta$ to make the inverter voltage lower and the DC current higher. The angle controlled by AMAX can be given by the formula below:

$$
A M A X=180-\beta
$$

Due to the current margin, the closed-loop current regulator at the inverter side will increase its trigger angle until the maximum value allowed. So the easiest way to let the inverter station run at a constant trigger angle is to set the AMAX angle as the output maximum limit of the current control angle.

\subsection{RAML and GAMMAO}

When the AC fault occurs to the rectifier, the angle $\alpha$ will rapidly decrease to the minimum value allowed. But when the system recovers, the DC current will grow too fast if the angle $a$ is very small. In order to prevent the situation above, we set a controller RAML. Its function is to set a minimum limit of angle a, RAML-ORD, which is usually 30 degrees when a lower AC voltage is measured. After the fault eliminated, RAML-ORD will decrease to 0 at a constant speed.

And in DC project, we also have inverter turn-off angle limit, GAMMA0. Its control logic is shown in figure 2 .

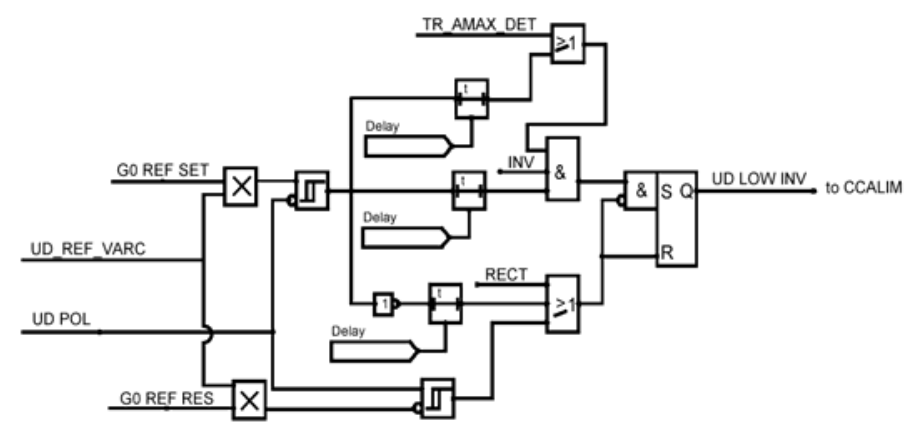

Figure 2 Control logic of GAMMA0

As we see above, when the DC voltage measured at the inverter side is smaller than the set value, G0_REF_SET, it will trigger UD_LOW_INV. When the ${ }^{-} \mathrm{DC}^{-}$voltage recovers to G0_REF_RES, UD_LOW_INV becomes 0 , and trigger angle turns back to the output of current controller. After the AC or DC fault is eliminated, this control strategy can maintain the trigger angle at a lager value, which will reduce the DC current recovery rate, prevent the current increase too fast and the system absorb too much reactive power. 


\subsection{Current command computation and voltage dependent current limit control}

For the control system in DC project, after the power instruction is given, current command computation will output the current instruction and import into voltage dependent current limit control (VDCOL). The input of current command computation is given through a filter. The filter time constant determines the difference between the input value and the DC current measured, and it will also determine DC system dynamic response to some extent.

On the other hand, when the DC voltage drops, VDCOL will decrease the current instruction. This control will keep the system stable when there are disturbances. After the AC fault is eliminated, VDCOL can make the system restart quicker, and prevent continuous commutation failure. The static characteristics of VDCOL is shown in fig. 3

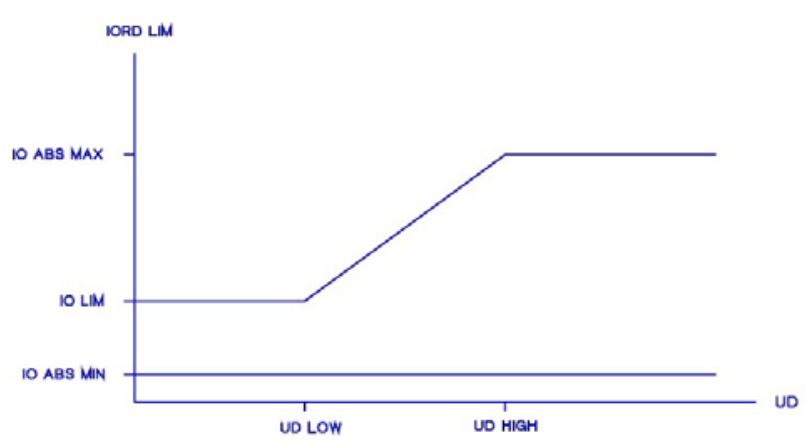

Figure 3. The static characteristics of VDCOL

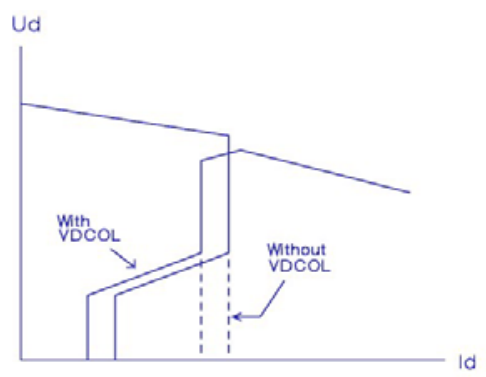

Figure 4. VDCOL's affection on $\mathrm{U}_{\mathrm{d}} / \mathrm{I}_{\mathrm{d}}$

The control logic of VDCOL is shown in figure 5.

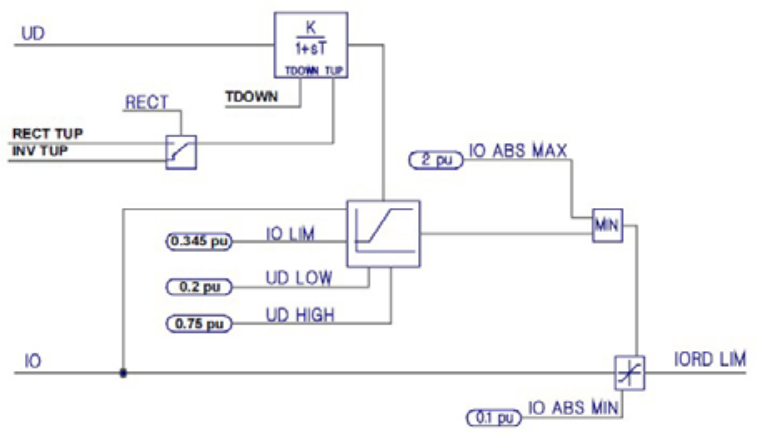

Figure 5. The control logic of VDCOL
As we seen above, VDCOL includes a minimum current limit(IO_ABS MIN). If the current is too low, it can prevent the SCR turn off the current. Usually, the value of IO_ABS_MIN is set as 0.1 p.u.. When the DC project is used for frequency control, it should be decreased to 0 . This allows the two commutation stations start at a small-load operation state. Also, there is a maximum current limit, which is usually equal to maximum overload limits.

\section{THE MAIN CONTROLLER'S IMPACT ON SYSTEM STABILITY}

As we know, there is a PI controller in the current controller. Scale factor and time constant determine the response of the current controller and DC system. The output is provided with upper and lower range. These limits limit the output of current controller in some special conditions. This limit is almost consistent with each DC project. So the parameters of the PI controller determine the dynamic response differences between the DC projects. Also, the voltage controller contains a PI controller. When it is in the operation of inverter, it will give a maximum value to the current controller to limit the max trigger angle output. When it runs as rectifier, it will serve as the lower limit of the current controller to limit the minimum value of trigger angle. The scale factor and time constant determine the response of voltage controller under fault conditions.

The key parameter of AMAX controller is I_GAIN, which determines the positive slope. If I_GAIN is too large, the trigger angle of AMAX will increase when the Dc current increase. And this will reduce the turn-off angle, which adversely affect the stable operation of the DC system.

While in RAML, there are three key parameters, AC voltage drop rate, trigger angle limit and trigger angle recovery rate. The smaller AC voltage drop rate is, the easier RAML starts. And when the trigger angle limit increases, the DC power recovery rate will drop. So these three parameters should be considered according to the AC system.

For GAMMA0 regulator, there are two main parameters, start set value and reset value, which determine the sensitivity of GAMMA0 to the DC voltage. The last is VDCOL. Its main function is maintaining the stability of the system when the disturbance appears or after it. The key parameters of VDCOL are DC voltage limit, DC current limit and filter time constant. Among them, the DC current and voltage limit directly determine the slope of the voltagecurrent characteristic curve of VDCOL and the recovery speed of the DC current. If we adjust the Dc voltage limit, we can directly change the response of VDCOL and even the operation state of the system. So these parameters should be set according to the recovery rate demand of the DC system. 


\section{CONCLUSION}

As mentioned above, we can conclude that:

Firstly, scale factor and time constant are the key parameters of current and voltage controller. They mainly affect the DC current response speed and overshoot.

Secondly, the slope of AMAX regulator determines the current-voltage characteristic curve.

Thirdly, these three regulators, RAML, GAMMA0 and VDCOL, they all have multiple key parameters. According to what we analyzed above, these parameters have great impact on the sensitivity to the disturbance, the stability of system and recovery rate after the $\mathrm{AC}$ or $\mathrm{DC}$ fault.

Above all, we should consider these parameters according to the state of the system, which include the recovery rate, the reactive power of $\mathrm{AC}$ system and so on.

\section{REFRENCES}

Chen Shuyong, Li Xinnian, Yu Jun, et al. A method based on the sin-cos components detection mitigates commutation failure in HVDC. Proceedings of the CSEE. Vol. 25 (2005):1-6 (in Chinese).

Lu Pengfei, LiXinnian, Chen Lingfang, et al. A new method of preventing commutation failure in HVDC based on sincoscomponents detection. International Conference on Electrical Engineering. YongPyong Korea: ICEE, 2006: 211.

Li Xinnian, Li Tao, Wang Jingfang. 2009. Impacts of $800 \mathrm{kV}$ DC power transmission from Yunnan to Guangdong on stability of China Southern Power Grid. Power System Technology. Vol. 33 (2009): 21-26 (in Chinese).

Li Xinnian, Wang Mingxin. Issues and measures of commutation failure in inverter station. Beijing: China Electric Power Research Institute, 2011.

Wu Ping, Lin Weifang, Sun Huadong. 2012. Research and electromechanical transient simulation on mechanism of commutation failure in multi-infeed HVDC power transmission system. Power System Technology. Vol. 36 (2012): 269-274 (in Chinese).

Wang Jingfang, Yu Jun, Li Xinnian, et al. Simulation and research of connected system in $800 \mathrm{kV}$ DC sending project. Beijing: China Electric Power Research Institute, 2005.

Wang Jingfang, Yu Jun, Li Xinnian, et al. PSCAD Calculation and research of multiple landing points DC sytem in China Southern Power Grid in 2010. Beijing: China Electric Power Research Institute, 2006. 